\section{Papulonecrotic tuberculid: report of a clinical case}

\author{
Ana Brinca, Pedro Andrade, \\ Ricardo Vieira, Américo Figueiredo \\ Dermatology Department, Hospitals of \\ Coimbra University, Portugal
}

\begin{abstract}
Papulonecrotic tuberculid is a rare, chronic, recurrent and symmetric eruption of necrotizing skin papules arising in crops, involving primarily the limbs. It occurs in less than $5 \%$ of active tuberculosis. Typically bacilli are not detected in any of the cutaneous lesions. Extracutaneous tuberculosis primary focus is only detected in $30-40 \%$ of the cases, being the lymph nodes the most common location. Antituberculosis treatment is rapidly effective and is often the main confirmation of the diagnosis.
\end{abstract}

\section{Case Report}

A 36-year-old previously healthy woman presented with a two-month history of recurrent asymptomatic multiple symmetric erythematous-to-violaceous papules and plaques, 0.5-3 $\mathrm{cm}$ in size, with central erosion or superficial crust in some of them, located mostly on the limbs, but also on the trunk and neck (Figure 1). Lesions gradually evolved into ulcers and then healed over a period of 3 weeks forming depressed scars. A firm asymptomatic mass nearly $2 \mathrm{~cm}$ wide was also noticed in the left axilla, compatible with an enlarged lymph node. Physical examination did not reveal any other significant changes. There was no recognized contact with tuberculosis patients and the patient denied fever, respiratory complaints, anorexia or weight loss. She complained of night sweats and diffuse myalgia and arthralgia going back to several weeks. Histopathologic analysis of a skin biopsy showed a dense granulomatous cell infiltrate with necrosis (Figure 2). Additionally, evidences of necrotizing granulomatous lymphadenitis consistent with the diagnosis of lymph node tuberculosis were observed on axillary lymph node biopsy. No significant changes were reported on several serum analyses apart from a raised erythrocyte sedimentation rate of $38 \mathrm{~mm}$ in the first hour. Total and differential white blood cell counts were normal. Blood Venereal Disease Research Laboratory (VDRL) test was non reactive and anti-human immnunodeficiency virus (HIV) serology was negative. Mycobacterium tuber- culosis was not identified on Ziehl-Neelsen staining or culture in all collected specimens (skin biopsy, axillary lymph node biopsy, peripheric blood and sputum). Chest X-ray and computed tomography were normal. The tuberculin skin test was strongly positive $(25 \mathrm{x} 30$ $\mathrm{mm}$ induration) with necrotic reaction. Based on the clinical features, positive tuberculin skin test and histopathologic findings, the diagnosis of papulonecrotic tuberculid (PNT) associated with tuberculous lymphadenitis was established and the patient was started on antituberculosis treatment (isoniazide $300 \mathrm{mg}$ daily, rifampicin $600 \mathrm{mg}$ daily and pyrazinamide $1500 \mathrm{mg}$ daily). Soon after anti-tuberculous therapy was started, remarkable clinical improvement was observed (Figure 3). Serologic evidence of hepatic toxicity (aspartate aminotransferase $=238 \mathrm{UI} / \mathrm{L}$ and alanine aminotransferase $=457 \mathrm{UI} / \mathrm{L}$ ) following two months on tuberculostatics determined the suspension of treatment for 40 days, without noticeable clinical deterioration. After normalization of hepatic function, the patient restarted isoniazide $300 \mathrm{mg}$ daily and pyrazinamide $1500 \mathrm{mg}$ daily associated with ciprofloxacin $750 \mathrm{mg}$ daily for a total of 12 months, with complete regression of active lesions and discrete residual scars and no recurrences for an oneyear follow-up.

\section{Discussion}

PNT is a rare condition, occurring predominantly in children and young adults. The majority of cases occur in patients infected with $M$. tuberculosis, but some cases have been reported after bacillus Calmette-Guérin (BCG) vaccination ${ }^{1}$. It occurs in less than $5 \%$ of active tuberculosis cases even in populations with a

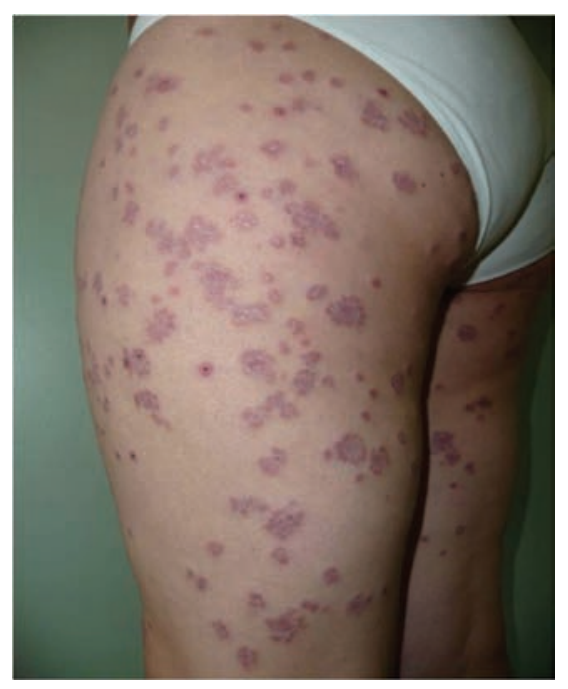

Figure 1. Involvement of inferior limbs.
Correspondence: Ana Brinca, Serviço de Dermatologia, Hospitais da Universidade de Coimbra, Praceta Mota Pinto, 3000-075 Coimbra, Portugal.

E-mail: anabrinca@gmail.com

Key words: papulonecrotic tuberculid; cutaneous tuberculosis.

Received for publication: 22 August 2011. Accepted for publication: 24 August 2011.

This work is licensed under a Creative Commons Attribution NonCommercial 3.0 License (CC BYNC 3.0).

(C) Copyright A. Brinca et al., 2011

Licensee PAGEPress, Italy

Dermatology Reports 2011; 3:e29

doi:10.4081/dr.2011.e29

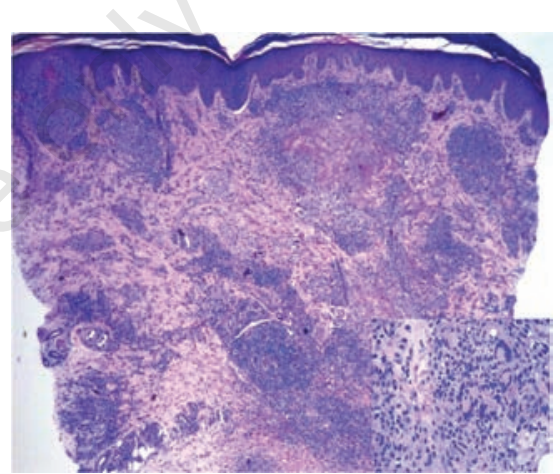

Figure 2. Dense granulomatous cell infiltrate with necrosis (hematoxylin \& eosin, $\times 40$; inset: $\times 200$ ).

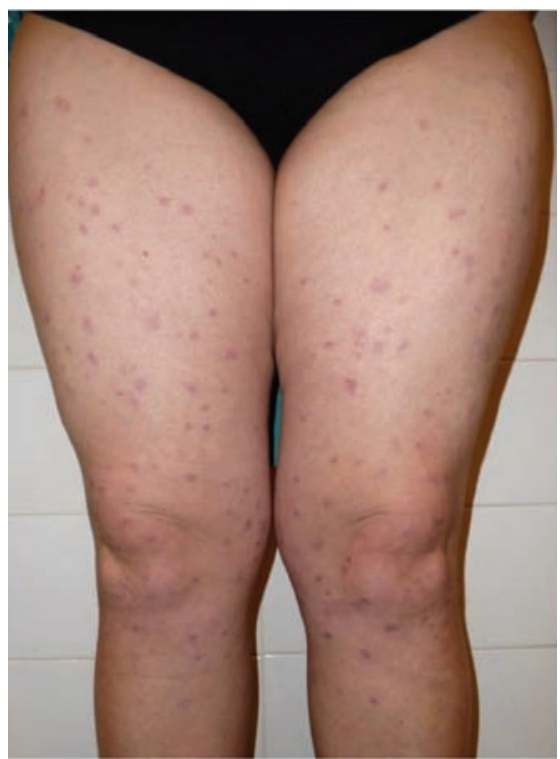

Figure 3. Clinical improvement after 2 weeks of treatment. 
high prevalence of tuberculosis. PNT is a chronic, recurrent and symmetric eruption of necrotizing skin papules arising in crops, involving primarily the limbs with a predilection for acral and extensor surfaces, rarely affecting the face. Circumscribed local involvement of the scalp ${ }^{2}$ and penis ${ }^{3}$ has also been described. Flares of new lesions can recur for many years if the condition is misdiagnosed.

Typically bacilli are not detected in any of the cutaneous lesions, since they result from the immunological expression of an internal focus of tuberculosis in an individual with a moderate-to-high degree of immunity. Nevertheless, Mycobacterial DNA has been identified in $77.8 \%$ of PNT skin biopsies using polymerase chain reaction technique ${ }^{4}$. Extracutaneous tuberculosis primary focus is only detected in $30-40 \%$ of the cases, being the lymph nodes the most common location. The tuberculin skin test is highly reactive in most patients, even though non reactive tests have been reported in patients with HIV infection ${ }^{5}$. Anti-tuberculosis treatment is rapidly effective and is often the main confirmation of the diagnosis. An active focus of tuberculosis should always be sought for and antituberculosis treatment promptly initiated.

\section{References}

1. Figueiredo A, Poiares-Baptista A, Branco M, et al. Papular tuberculids post-BCG vaccination. Int J Dermatol 1987;26:291-4.
2. Niemeyer-Corbellini JP, Spinatto D Boechat N, et al. Papulonecrotic tuberculid on the scalp. Int J Derm 2008;47:1028-32.

3. Padmavathy L, Chockalingam K, Rao LL, Ethirajan N. Recurrent, scarring penile ulcers. Papulonecrotic tuberculide. Indian J Dermatol Venereol Leprol 2006;72:86.

4. Jordaan HF, Van Niekerk DJT, Louw M. Papulonecrotic tuberculid. A clinical, histopathological and immunohistochemical study of 15 patients. Am J Dermatopathol 1994;16:474-85.

5. Friedman PC, Husain S, Grossman ME. Nodular tuberculid in a patient with HIV. J Am Acad Dermatol 2005;53(2 Suppl. 1): S154-6. 